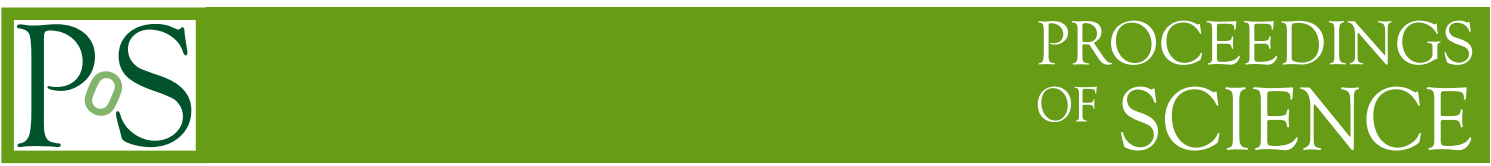

\title{
A 100 Msec INTEGRAL exposure of Bright Infrared Galaxies
}

\author{
R. Walter* \\ INTEGRAL Science Data Centre, Observatoire de Genève, Université de Genève, Chemin \\ d'Ecogia 16, CH-1290 Versoix, Switzerland \\ E-mail: Roland.Waltereunige.ch \\ N. Cabral \\ Université de Bordeaux 1, UFR de Physique, 351 Cours de la Libération, F-33405 Talence \\ Cedex, France
}

\begin{abstract}
The synthesis of the cosmic X-ray background requires a large population of Compton thick Active Galactic Nuclei that has not been detected so far. We probe whether bright infrared galaxies could arbor a population of Compton thick nuclei and if they could contribute significantly. We analysed 100 Msec of INTEGRAL observations obtained on 613 galaxies from the IRAS Revised Bright Galaxy Sample. We derived the average hard X-ray emission of Seyfert and various non Seyfert galaxy subsamples to estimate their contribution to the hard X-ray background. The Seyfert $1 \& 2$ are clearly detected at hard X-rays. None of the other galaxy subsamples is detected. The upper limit obtained for non Seyfert galaxies is as low as $7 \times 10^{-13} \mathrm{erg} \mathrm{s}^{-1} \mathrm{~cm}^{-2}$. ULIRGs are at least 5 times under-luminous at hard X-rays when compared to Seyferts. On average non Seyfert galaxies do not contain active nuclei brighter than $10^{41} \mathrm{erg} / \mathrm{s}$ at hard X-rays. $64 \%$ of the hard X-ray emission from the sample originates in absorbed active nuclei. Non-Seyfert galaxies contribute for less than $7 \%$ and do not arbor the Compton thick nuclei required to synthesize the X-ray background.
\end{abstract}

7th INTEGRAL Workshop

September 8-11 2008

Copenhagen, Denmark

${ }^{*}$ Speaker. 


\section{Introduction}

While accreting super-massive black-holes are believed to power Active Galactic Nuclei (AGN; 1), the apparent spectral energy distribution of these objects depends on many physical parameters such as the black-hole mass and accretion rate, the nature of the accretion flow, the inclination on the line of sight and the intrinsic obscuration.

The hard X-ray background, with a prominent maximum around $30 \mathrm{keV}$, requires that most AGNs are obscured (2). Quantitatively, population synthesis models indicate that 60 to $80 \%$ of all AGNs should be obscured and that about $50 \%$ of these should be Compton thick (3). Nuclear starbursts in the central few parsecs of a galaxy could provide the necessary obscuring material (4) to explain a large population of Compton thick objects. These nuclear starbursts also link stellar formation and the black-hole activity $(5 ; 6)$.

Various techniques have been used to study the relation between starbursts and AGN activity, based on the study of the infrared spectral energy distribution (7) or on correlations between infrared and X-ray or radio detections $(8 ; 9)$. As hard X-ray emission is an ubiquitous signature of AGN activity (10) and as it is insensitive to photoelectric absorption, the correlation between infrared and hard X-ray fluxes is an interesting probe for the existence of highly absorbed AGNs.

Even if a fraction of the AGN population features various signature of absorption - like an absence of broad emission lines, scattered emission (11), reddening (12; 13), or soft X-ray photoelectric absorption $(14)$ - recent low sensitivity hard X-ray surveys $(15 ; 16)$ have failed to detect a population of Compton thick objects.

In order to improve the sensitivity of the current hard X-ray surveys by one order of magnitude, we stacked the hard X-ray signal measured by INTEGRAL for various types of infrared bright galaxies to check their contribution to the hard X-ray background. The sample of sources is presented in section 2, the INTEGRAL data and analysis is described in section 3 and their correlation with infrared data is discussed in section 4.

\section{Source sample}

In 1983, the Infrared Astronomical Satellite (IRAS) surveyed approximately $96 \%$ of the sky in four broad wavelenght bands centered on 12, 25, 60 and $100 \mu \mathrm{m}$. The IRAS Point Source Catalog (PSC) and the IRAS Faint Source Catalog (FSC) were produced listing 245889 and 173044 infrared sources respectively (the PSC extends to lower galactic latitude than the FSC). IRAS discovered the luminous and ultra-luminous infrared galaxies (LIRGs and ULIRGs) in the local Universe with infrared luminosities larger than $10^{11} \mathrm{~L}_{\odot}$ and $10^{12} \mathrm{~L}_{\odot}$ respectively.

(17) used the IRAS FSC and PSC to constitute the IRAS Revised Bright Galaxy Sample (RBGS). The RBGS is a complete flux-limited sample of all extragalactic objects brighter than 5.24 Jy at $60 \mu \mathrm{m}$, covering the entire sky surveyed by IRAS at galactic latitudes $|\mathrm{b}|>5^{\circ}$. The median redshift of the RBGS galaxies is $\mathrm{z}=0.0082$ and their luminosity function breaks at $L_{i r} \sim 10^{10.5} L_{\odot}$ from a slope of -0.6 to -2.2 .

Among the 629 objects of the RBGS, we excluded the SMC and LMC because they are extended for the imager on board INTEGRAL, 13 objects because of their proximity with other 
sources detected at hard X-rays and NGC 6240, the complex ULIRG and Compton thick double Seyfert nucleus (18), detected by INTEGRAL as well.

The resulting sample of 613 objects was classified in six sub-samples according to their object type defined by SIMBAD: 93 Seyfert galaxies (including 14 Seyfert 1 and 64 Seyfert 2) and 520 "non Seyfert" galaxies, missing clear signature for an active nucleus, including 12 ULIRGs $\left(\mathrm{L}_{60 \mu \mathrm{m}} / \mathrm{L}_{\odot}>10^{12}\right), 144 \mathrm{LIRGs}\left(10^{12}>\mathrm{L}_{60 \mu \mathrm{m}} / \mathrm{L}_{\odot}>10^{11}\right)$ and 41 Liners.

\section{INTEGRAL data and analysis}

All public data obtained by the INTEGRAL soft $\gamma$-ray imager IBIS/ISGRI $(19 ; 20)$ until January 2007 are considered in this study. The INTEGRAL imager has a large field of view of $29^{\circ}$ square with a spatial resolution of 12 arcmin. Several thousands pointings including at least one source of the sample in its field of view and with an exposure longer than $120 \mathrm{~s}$ were selected, spanning times between Dec. 30, 2002 (revolution 26) and Jan. 17, 2007 (revolution 520).

The ISGRI data were reduced using the INTEGRAL Offline Scientific Analysis software version 7.0 publicly released by the INTEGRAL Science Data Centre (21). Individual sky images for each pointing have been produced in a broad energy band of $18-80 \mathrm{keV}$. Only catalogued sources with significance above 5 have been used for image cleaning with source position fixed when known accurately.

To measure the average hard X-ray properties of the selected galaxy subsamples we have built mosaic images of all subsample sources modifying the coordinate system of each individual image such that the coordinates of each subsample sources were set to an arbitrary fixed position $(\alpha=0$, $\delta=0$ ). The resulting image provides a stack of all ISGRI data available on all sources of interrest.

To build the best possible image we have excluded 1455 individual images (many before revolution 38) which had background fluctuations larger than 1.1 in the significance map and 779 images with minimum significance lower than -5.5. 36030 images were finally included in the processing (some of them many times, when including several sources).

The 500x500-pixels mosaic images were built in equatorial coordinates centerred on $(\alpha=0$, $\delta=0$ ) with a tangential projection using a factor two over-sampling when compared to the individual input sky images; this results in a pixel size of 2.4 arcmin in the center of the mosaic. The photometric integrity and accurate astrometry are obtained by calculating the intersection between input and output pixels and weighting count rates according to the overlapping area.

The effective exposure obtained at the center of the mosaics are between 1.7 and $96.0 \mathrm{Ms}$ for the various galaxy subsamples. To investigate the quality of the mosaics, we studied the distribution of the pixelsÕ significance, which is expected to be Gaussian with average 0 and dispersion 1 . We fitted a Gaussian distribution to the part of the histogram with $\sigma \leqslant 3$ to avoid the strong positive tail due to real sources. While the width of the pixel distributions $(\approx 1.2)$ are not unusual the centroid of the distribution is significantly displaced towards positive values for the sample of "non Seyfert" galaxies where the effective exposure reaches almost $100 \mathrm{Ms}$. Our analysis shows that the individual ISGRI images appear slightly positive on average, which could not be detected with typical exposures up to several Ms. The correction of that systematic effect is out of the scope of the present study. 
Table 1: Average signal (or $5 \sigma$ upper limit) detected for the various subsamples at hard X-rays (18-80 keV) and at $60 \mu \mathrm{m}$. Effective hard X-ray exposure, count rates and integrated flux are provided together with the average $60 \mu \mathrm{m}$ flux.

\begin{tabular}{lrccc}
\hline \hline Sample & $\begin{array}{r}\text { Exp. } \\
\mathrm{Ms}\end{array}$ & $\begin{array}{c}\text { Count rate } \\
\mathrm{ct} / \mathrm{s}\end{array}$ & $\begin{array}{c}\mathrm{F}_{18-80 \mathrm{keV}} \\
\mathrm{erg} / \mathrm{s} \mathrm{cm}^{2}\end{array}$ & $\begin{array}{c}\mathrm{F}_{60 \mu \mathrm{m}} \\
\mathrm{Jy}\end{array}$ \\
\hline Seyfert 1 & 1.7 & $2.25 \pm 0.02$ & $1.28 \cdot 10^{-10}$ & 18.3 \\
Seyfert 2 & 12.1 & $0.87 \pm 0.01$ & $0.49 \cdot 10^{-10}$ & 31.1 \\
Seyfert & 16.1 & $1.04 \pm 0.01$ & $0.59 \cdot 10^{-10}$ & 28.9 \\
\hline ULIRGs & 4.1 & $<0.06$ & $<3.4 \cdot 10^{-12}$ & 10.2 \\
Liners & 7.5 & $<0.045$ & $<2.6 \cdot 10^{-12}$ & 29.6 \\
LIRGs & 26.7 & $<0.02$ & $<1.1 \cdot 10^{-12}$ & 12.9 \\
non Seyfert & 96.0 & $<0.013$ & $<7.4 \cdot 10^{-13}$ & 19.1 \\
\hline
\end{tabular}

A positive average signal is clearly detected in the mosaics built for the three Seyfert subsamples. No average excess is detected for the other galaxy subsamples. As expected, the position $(\alpha=0, \delta=0)$ is well within the $90 \%$ error box corresponding to the excesses found for the three Seyfert subsamples and the $1 \sigma$ width of these excesses are between 5.7 and 6.0 arcmin, in good agreement with the ISGRI point spread function.

Table 1 lists the average signal (or $5 \sigma$ upper limits) extracted at hard X-rays from each ISGRI mosaic with mosaic_spec (corrected for the higher sky background systematics obtained for the large "non Seyfert" galaxy subsample) together with the average flux measured by IRAS at $60 \mu \mathrm{m}$ (17) for each subsample. The hard X-ray fluxes were derived from the count rates using a spectral index of $\Gamma=1.8$. The hard X-ray detections obtained for the three Seyfert subsamples are more than one order of magnitude brighter than the upper-limits obtained for the other galaxy subsamples with comparable effective exposures. The hard X-ray upper limit obtained for the "non Seyfert" subsample is a hundred times less than the flux detected in average from the Seyfert galaxies.

\section{Discussion}

\subsection{Average spectral energy distribution}

In order to compare the average spectral energy distribution of the various galaxy subsamples we normalized their spectra at $60 \mu \mathrm{m}$, where most of the emission is believed to originate from molecular clouds or diffuse dust reprocessing stellar light (22).

The ratios between the flux densities at hard X-rays and at $60 \mu \mathrm{m}$ are $12 \%$ for Seyfert 1, 3\% for Seyfert 2, below $0.6 \%$ for ULIRGs, below $0.1 \%$ for the LIRGs and Liners, and finally below $0.07 \%$ for the "non Seyfert" galaxies all together.

From the hard X-ray point of view we find a clear separation between the average Seyfert dominated and non Seyfert galaxies, the latter being not detected. As the hard X-rays are not affected by moderate absorption this difference could be intrinsic to the source samples and/or signature of Compton thick absorption in the galaxy subsamples lacking Seyfert signatures. 
The ratio between the flux densities obtained at hard X-rays from high-mass X-ray binaries and in the far infrared can be estimated from (23) and (24) and is not larger than $0.03 \%$. This is two orders of magnitude lower than the hard X-ray signal obtained for the Seyfert subsamples and still below the upper limits obtained for the "non Seyfert" galaxies. Our upper limits are therefore consistant with the hard X-ray emission expected from stellar activity.

The Seyfert 2 are 4.5 times more numerous than the Seyfert 1 in the RGBS in agreement with the results of (25) and (26) for a sample dominated by low luminosity sources. Their average hard $\mathrm{X}$-ray luminosity, when normalized to the infrared, is consequently also lower.

The hard X-ray upper limit obtained for the ULIRGs indicate that, on average, these sources emit less than $20 \%$ of the emission detected in Seyferts. This is consistent with the fact that less than $30 \%$ of the ULIRGs arbor a significant $(>10 \%)$ AGN contribution in the far infrared $(27 ; 22)$ and with the predominance of their starburst emission even at soft X-rays (28). Note that as the number of nearby ULIRGs is small, our upper limits are an order of magnitude above the extrapolation at hard X-rays of the XMM-Newton detections.

Active nuclei absorbed by Compton thick material in ULIRGs or LIRGs could, in principle, remain completely hidden at hard X-rays. Even in such a case, the reprocessed AGN emission would not contribute much more than $10 \%$ of the far infrared flux and could also remain hardly detectable there. To accomodate our hard X-ray upper limits, no more than 3 and 20\% of the hard X-ray emission should be transmitted on average in LIRGs and ULIRGs respectively. For the ULIRGs, this means that active nuclei, if they exist, should be obscured by $\mathrm{N}_{\mathrm{H}}>10^{24} \mathrm{~cm}^{-2}$ and that no more than a few of them in the 12 sources sample should remain unabsorbed. For the LIRGs, the active nuclei, if present, should be obscured by $\mathrm{N}_{\mathrm{H}}>5 \times 10^{24} \mathrm{~cm}^{-2}$ and no more than a few out of 144 should be unobscured.

The "non Seyfert" galaxies are, on average, very faint at hard X-rays. The upper limit we have obtained indicates that their hard X-ray emission is not driven by active nuclei brighter than $\mathrm{L}_{\mathrm{X}} \sim 10^{41} \mathrm{erg} / \mathrm{s}$ on average. The presence of bright active nuclei absorbed by $\mathrm{N}_{\mathrm{H}}>5 \times 10^{24} \mathrm{~cm}^{-2}$ with no more than 15 unobscured ones out of 520 sources can however not be ruled out.

The hard X-ray upper limit obtained for the Liner galaxies is in agreement with active nuclei or starbursts of low X-ray luminosity $\left(\sim 10^{40} \mathrm{erg} / \mathrm{s}\right)$ reported in these objects (29).

\subsection{Contributions to the hard X-ray background}

We can estimate the relative contributions of the non-Seyfert and Seyfert galaxies from the RBGS to the hard X-ray background (CXB). The cumulative hard X-ray flux of the latter is $1.8 \times$ $10^{-9}$ and $3.1 \times 10^{-9} \mathrm{erg} \mathrm{s}^{-1} \mathrm{~cm}^{-2}$ for the Seyfert 1 and Seyfert 2 respectively (slightly less than $1 \%$ of the total CXB). The cumulative hard X-ray flux of the non Seyfert galaxies is $<0.38 \times 10^{-9}$ $\mathrm{erg} \mathrm{s}^{-1} \mathrm{~cm}^{-2}$.

The ratio of the contributions of the non Seyfert galaxies and of the Seyfert 1 galaxies in the RGBS is $<0.21$ (NGC 4303 is the only Seyfert galaxy listed in table ??). The ratio of the contributions of the RBGS Seyfert 2 and Seyfert 1 to the hard X-ray background is 1.7.

Assuming that Seyfert 1 and 2 are optically thin and, respectively, mildly absorbed (which is not completely accurate in general; e.g. 30; 31), the fraction of the CXB emitted at hard X-rays by absorbed objects $\left(\mathrm{N}_{\mathrm{H}}>10^{22} \mathrm{~cm}^{-2}\right)$ is $64 \%$ and the fraction emitted by potentially Compton thick non Seyfert objects is $<7 \%(5 \sigma)$. 
These fractions can be compared to the predictions of CXB synthesis models and in particular to these of (3), which could be obtained through the on-line tool "POMPA". Our sample is flux limited in the infrared, the corresponding X-ray flux limit for an individual Seyfert galaxy is of the order of $10^{-11} \mathrm{erg} \mathrm{s}^{-1} \mathrm{~cm}^{-2}$. For such a sample the CXB synthesis model indicates that absorbed and Compton thick sources should contribute for $65 \%$ and, respectively, $18 \%$ of the CXB at 30 $\mathrm{keV}$.

The fraction of the CXB to be accounted for by absorbed sources agrees very well with the results of our observations. The fraction expected to be emitted by Compton thick sources is however significantly larger than the upper limits obtained for non-Seyfert galaxies. Non Seyfert galaxies are therefore unlikely to arbor Compton thick active nuclei contributing significantly to the CXB.

\section{Conclusions}

We have derived the most sensitive average hard X-ray fluxes for subsamples of Seyfert and non Seyfert galaxies stacking INTEGRAL/ISGRI images of 613 members of the IRAS Revised Bright Galaxy Sample.

The Seyfert $1 \& 2$ subsamples are clearly detected at hard X-rays while none of the other galaxy subsamples is detected.

The upper limit obtained on the ULIRGs indicates that on average these object emit less than $20 \%$ of the average Seyfert emission at hard X-rays. This is consistant with previous results suggesting that the majority of these objects do not arbor significant AGN activity.

On average, "non Seyfert" galaxies do not contain any active nuclei brighter than $10^{41} \mathrm{erg} / \mathrm{s}$ at hard X-rays. If they arbor Compton thick AGNs, these should be highly absorbed $(>5 \times$ $10^{24} \mathrm{~cm}^{-2}$ ) and no more than a few $\%$ of them could remain unabsorbed.

The RGBS accounts for less than $1 \%$ of the CXB. $64 \%$ of this is produced by absorbed ( $>$ $10^{22} \mathrm{~cm}^{-2}$ ) sources, in very good agreement with CXB synthesis models. Non Seyfert galaxies contribute for less than $7 \%$ and have not been detected statistically. These galaxies are unlikely to arbor the Compton thick AGNs required to synthesize the CXB. Local Compton thick AGNs, as a population, should therefore be looked for among Seyfert 2 or infrared weak galaxies.

\section{References}

[1] Lynden-Bell, D., Nature 223 (1969) 690.

[2] Setti, G. and Woltjer, L., A\&A 224 (1989) L21.

[3] Gilli, R., Comastri, A., and Hasinger, G., A\&A 463 (2007) 79.

[4] Ballantyne, D. R., ArXiv e-prints 806 (2008).

[5] Watabe, Y., Kawakatu, N., and Imanishi, M., ApJ 677 (2008) 895.

[6] Kawakatu, N. and Wada, K., ApJ 681 (2008) 73.

[7] Genzel, R. et al., ApJ 498 (1998) 579. 
[8] Barger, A. J. et al., AJ 129 (2005) 578.

[9] Polletta, M. d. C. et al., ApJ 642 (2006) 673.

[10] Mushotzky, R. F., Done, C., and Pounds, K. A., ARA\&A 31 (1993) 717.

[11] Schmidt, G. D., Smith, P. S., Hines, D. C., Tremonti, C. A., and Low, F. J., ApJ 666 (2007) 784.

[12] Walter, R. and Fink, H. H., A\&A 274 (1993) 105.

[13] Wilkes, B. J. et al., ApJ 634 (2005) 183.

[14] Tozzi, P. et al., A\&A 451 (2006) 457.

[15] Paltani, S. et al., A\&A 485 (2008) 707.

[16] Tueller, J. et al., ApJ 681 (2008) 113.

[17] Sanders, D. B., Mazzarella, J. M., Kim, D.-C., Surace, J. A., and Soifer, B. T., AJ 126 (2003) 1607.

[18] Vignati, P. et al., A\&A 349 (1999) L57.

[19] Ubertini, P. et al., A\&A 411 (2003) L131.

[20] Lebrun, F. et al., A\&A 411 (2003) L141.

[21] Courvoisier, T. J.-L. et al., A\&A 411 (2003) L53.

[22] Vega, O. et al., A\&A 484 (2008) 631.

[23] Grimm, H.-J., Gilfanov, M., and Sunyaev, R., MNRAS 339 (2003) 793.

[24] Kennicutt, Jr., R. C., ApJ 498 (1998) 541.

[25] La Franca, F. et al., ApJ 635 (2005) 864.

[26] Akylas, A., Georgantopoulos, I., Georgakakis, A., Kitsionas, S., and Hatziminaoglou, E., A\&A 459 (2006) 693.

[27] Armus, L. et al., ApJ 656 (2007) 148.

[28] Franceschini, A. et al., MNRAS 343 (2003) 1181.

[29] González-Martín, O., Masegosa, J., Márquez, I., Guerrero, M. A., and Dultzin-Hacyan, D., A\&A 460 (2006) 45.

[30] de Rosa, A. et al., A\&A 483 (2008) 749.

[31] Brightman, M. and Nandra, K., ArXiv e-prints 808 (2008). 